Revue d'histoire de l'Amérique française

RAS REVUE D.HISTOIRE DE L'AMÉRIQUE FRANÇAISE

\title{
Le métahistorien Arnold J. Toynbee et la minorité canadienne-française
}

\section{Michel Brunet}

Volume 5, numéro 3, décembre 1951

URI : https://id.erudit.org/iderudit/801716ar

DOI : https://doi.org/10.7202/801716ar

Aller au sommaire du numéro

Éditeur(s)

Institut d'histoire de l'Amérique française

ISSN

0035-2357 (imprimé)

1492-1383 (numérique)

Découvrir la revue

Citer cet article

Brunet, M. (1951). Le métahistorien Arnold J. Toynbee et la minorité canadienne-française. Revue d'histoire de l'Amérique française, 5(3), 362-372. https://doi.org/10.7202/801716ar d'utilisation que vous pouvez consulter en ligne.

https://apropos.erudit.org/fr/usagers/politique-dutilisation/ 


\title{
LE MÉTAHISTORIEN ARNOLD J. TOYNBEE ET LA MINORITÉ CANADIENNE-FRANÇAISE
}

\begin{abstract}
Arnold J. Toynbee jouit d'un grand prestige. Il figure parmi les intellectuels dont notre monde contemporain s'enorgueillit. Sa vaste enquête sur la naissance, le développement, la décadence et la mort des civilisations ${ }^{1}$ lui a révélé l'importance du sentiment religieux dans l'évolution historique de l'humanité. Il s'est graduellement convaincu que, si nous voulons étudier l'histoire dans son entité, nous devons "reléguer l'histoire économique et politique au second plan et donner à l'histoire religieuse la primauté. Car la religion, après tout, est l'affaire sérieuse de la race humaine."'2 $M$. Toynbee prévoit - un métahistorien devient facilement prophète - que d'ici trois mille ans l'unité de tous les hommes se sera réalisée non pas à cause des nécessités de la technique et de l'économie, encore moins par la guerre et par la politique, mais par la religion. ${ }^{3}$ Honnêtement, comme tout chercheur consciencieux à qui ses recherches imposent une conclusion, il a affirmé sa foi en l'œuvre providentielle de l'Église catholique qu'il représente cornme l'héritière de toutes les autres Églises et de toutes les civilisations. ${ }^{4}$ Ce témoignage spontané d'un Anglais de tradition libérale, élevé dans l'Église d'Angleterre, prend un sens tout particulier au moment même où l'Église
\end{abstract}

1. Arnold J. Toynbee, A Study of History (6 vol., Londres, New York et Toronto, 1948); édition abrégée de D.C. Somervelle, A Study of History (New York, 1947). Les six volumes de A Study of History parurent d'abord de 1934 à 1939.

2. Arnold J. Toynbee, Civilization on Trial (New York, 1948), 94.

3. Ibid., 216. Un récent article de M. Toynbee laisse croire que l'auteur a modifié son point de vue sur la révolution industrielle du XXe siècle. Il ne ménage plus son admiration pour les réalisations de la technique et annonce un âge d'abondance pour les peuples qui vivent encore dans une économie agricole arriérée. Voir "Not the Age of Atoms But of Welfare for All", New York Times Magazine, 21 octobre 1951.

4. Voir sa conférence "Christianity and Civilization", dans ibid., 225-252. Consulter aussi Study of History (édition abrégée), 359. 
romaine subit de la part de ses ennemis un assaut peut-être plus formidable que tous ceux qui lui ont été livrés au cours de sa longue histoire. Tous les catholiques seront reconnaissants envers M. Toynbee de n'avoir pas hésité à faire cette profession de foi.

Les œuvres érudites de M. Toynbee ne l'empêchent pas d'être un écrivain très populaire ${ }^{5}$. Ses livres ont une grande vogue. Des milliers de personnes ont suivi ses nombreuses conférences aux Etats-Unis et en Angleterre. La radio lui a permis d'atteindre de très vastes auditoires. Cette popularité d'un écrivain et d'un érudit, n'ayant rien d'un auteur de romans à sensation ou d'un manufacturier de romans-feuilletons du XIXe siècle, est tout à l'honneur de notre monde contemporain. Ce fait mérite l'attention des contempteurs du XXe siècle et devrait les réconcilier avec lui. Si des milliers de lecteurs d'Amérique et d'Europe lisent et discutent les œuvres de Toynbee, c'est parce que les hommes ont gardé, même à l'âge de la machine et de la technique, leurs aspirations vers l'idéal, le beau, le bien, le vrai. Les pessimistes qui méprisent systématiquement le siècle dans lequel nous vivons, ignorent trop facilement les preuves - elles sont nombreuses - qui viennent en contradiction avec la thèse selon laquelle notre âge serait irrémédiablement condamné au matérialisme le plus abject. Tout un arsenal de clichés nous cache la vérité sur un siècle qui n'est peut-être pas meilleur que ceux qui l'ont précédé mais qui n'est pas pire non plus.

Toute popularité demande sa rançon. Les disciples rendent généralement un mauvais service à leur maître. M. Toynbee compte de nombreux admirateurs qui lui reconnaissent une autorité d'historien. Les plus enthousiastes vont jusqu'à soutenir que A Study of History classe son auteur parmi les plus grands historiens de notre époque, sinon le plus grand ${ }^{6}$. Toynbee a su se gagner et conserver la sympathie de ses lecteurs et de ses auditeurs par l'élévation de sa pensée, la puissance de son imagination, ses dons littéraires et l'audace de ses hautes spéculations. Ses critiques les plus sévères rendent hommage au talent de l'écrivain, à son érudition phénoménale et à l'ori-

5. Une grande revue d'information, tirée à plus d'un million d'exemplaires chaque semaine, lui a consacré un long article. Voir Times, édition du 17 mars 1947.

6. Voir opinion de Richard Arès, "Sur une prédiction d'Arnold Toynbee", Relations, 10 (Avril 1950): 93. 
ginalité fascinatrice de quelques-unes de ses idées et de ses hypothèses. ${ }^{7}$ Cependant, ses fidèles exagèrent lorsqu'ils le consacrent historien.

Son œuvre principale, $A$ Study of History, n'est pas de l'histoire. Personne ne nie à $M$. Toynbee le droit de chercher à découvrir des constantes par la méthode empirique. Il conserve la liberté de s'aventurer dans des voies nouvelles. L'historien de métier les redoute et les évite parce qu'il sait que les sciences historiques ne sont pas suffisamment avancées pour en arriver à établir des lois qui expliqueraient infailliblement le comportement des hommes vivant en société. L'étude de $M$. Toynbee est prématurée à cause de son ampleur et n'appartient pas au domaine que cultive l'historien véritable. ${ }^{8}$

L'historien est beaucoup plus modeste que M. Toynbee. Il reconnaît la faiblesse de ses moyens d'investigation. Sa tâche essentielle consiste à "présenter un ensemble de faits qu'il a pu vérifier" après s'être demandé: "Qu'est-ce qui s'est réellement produit et pourquoi ?", selon l'un des maitres contemporains de l'historiographie américaine. ${ }^{\circ}$ L'historien doit savoir que "les causes, en histoire pas plus qu'ailleurs, ne se postulent pas. Elles se cherchent..." ${ }^{10} \mathrm{Au}$ cours de ses recherches, il se soumet scrupuleusement à la dure discipline que lui impose la méthodologie de la science historique. Il est tenu aux exigences de la loi de la preuve. S'il a recours, comme le savant de laboratoire, aux hypothèses, il procède avec une infinie précaution. Lorsqu'il réfléchit sur les faits et sur les événements que sa recherche lui a révélés, il s'assure que celle-ci a été objective et complète. Quand il use de son droit - il lui est loisible de ne pas s'en prévaloir - de porter un jugement sur le passé et de donner ses conclu-

7. Voir Pierre Gourou, "Civilisations et malchance géographique", Annales: Economies, Societés, Civilisation, 4 (Octobre 1949): 450; Frank H. Underhill, "Arnold J. Toynbee, Metahistorian", Canadian Historical Review, 32 (Septembre 1951): 218; Pitirim A. Sorokin, "Arnold 'J. Toynbee's Philosophy of History", Journal of Modern History, 12 (1940): 374-379.

8. Voir opinion de Georges Lefebvre, "Compte rendu critique de A.J. Toynbee, "A Study of History", Revue historique, 201 (Janvier 1949): 109-113.

9. Samuel Eliot Morison, "Faith of a Historian", American Historical Review, 56 (Janvier 1951): 263.

10. Marc Bloch, A pologie pour l'historien (Paris, 1949), 103. 
sions personnelles, il doit faire preuve d'honnêteté intellectuelle, d'esprit scientifique et d'humilité.

M. Toynbee ne partage pas la même prudence et n'éprouve pas les mêmes scrupules. Il utilise l'histoire avec l'intention évidente de ne pas se soumettre aux bornes que l'état actuel des recherches impose à ceux qui se chargent de renseigner les hommes sur leur passé. Ces écrivains, ces dissertateurs ouvrent peut-être de nouveaux horizons en faisant œuvre de pionniers. "Sans aucun doute, ils rendent de grands services aux historiens et aux sociologues ${ }^{12}$, mais ils n'ont pas le droit de se considérer ou d'être considérés comme des historiens, même si l'histoire leur fournit les matériaux sur lesquels ils travaillent. Ils poursuivent un but différent de celui de l'historien et on a créé pour eux les mots métahistoire et métahistorien. ${ }^{13}$

Lè métahistorien prétend, en quelque sorte, au rôle de prophète et de divin. Entreprise ambitieuse et tentante. Celui qui s'y hasarde aurait profit à méditer cette phrase de Jacques Maritain: "Et quant à discerner les causes et les lois suprêmes en jeu dans le cours des événements, il nous faudrait, pour le faire avec certitude, être du conseil du souverain Plasmateur, ou directement éclairé par lui."14 Le philosophe français avoue que "livrer aux hommes la philosophie de leur histoire est un office proprement prophétique." 15 L'historien, même celui qui reconnaît ne pouvoir écrire l'histoire sans croire à des normes, se refuse à jouer un tel rôle. S'il l'accepte, il ne fait plus de l'histoire.

M. Toynbee métahistorien et prophète échappe partiellement à la censure des historiens. Ceux-ci, cependant, se réservent le privilège de le tenir comptable de ses erreurs lorsqu'il envahit le domaine propre à l'histoire. Tous ses critiques ont constaté que, pour étayer ses théories sur l'origine, l'épanouissement et la décadence des civilisations, il ne choisit que les faits qui cadrent avec ses préconcep-

11. Voir plaidoyer d'un défenseur de Toynbee, G.A. Birks, "Toynbee and His Critics", Philosophy, 25 (Octobre, 1950): 336-340.

12. Lefebvre, "Compte rendu critique", loc. cit., 112; Gourou, "Civilisations et malchance géographique", loc. cit., 450.

13. Voir Allan Bullock, "The Historian's Purpose: History and Metahistory", History Today, 1 (Février 1951): 5-11.

14. Jacques Maritain, Tois Reformateurs (Paris, 1925), 131-132.

15. Ibid., 132. 
tions. ${ }^{16}$ C'est la faute commune à tous ceux qui défendent un système ou croient en un déterminisme historique quelconque. Lorsque l'auteur de $A$ Study of History aborde l'analyse d'un problème historique, on sent que son siège est fait. D'avance, il a tout prévu. Le lecteur se laisse gagner par l'érudition, l'assurance, la virtuosité, l'éloquence du dissertateur. Celui-ci est lui-même le premier à se convaincre de la justesse de ses vues.

C'est ici que l'historien a le droit d'intervenir et de mettre le métahistorien et ses lecteurs garde. La majorité des historiens contemporains a renoncé aux illusions de l'école positiviste qui prétendait donner du passé une image absolument fidèle, scientifique. "Personne aujourd'hui, remarque Henri-Irénée Marrou, n'oserait plus réduire le rôle de l'historien à celui d'un appareil enregistreur, simplement chargé de reproduire avec une fidélité mécanique son "objet", le passé, à le photographier, comme on eat dit vers 1900 (l'image eât été trompeuse, car nous avons appris à mieux nous servir de nos machines et nous savons aujourd'hui tout ce qu'a de "construit" une image photographique qui transcrit le réel en choisissant et déformant suivant le point de vue, la profondeur du champ, la mise au point, etc.)."17 L'historien concède qu'il lui est matériellement impossible de donner à ses lecteurs tous les faits qu'il a recueillis. Il doit nécessairement faire un choix. Celui-ci ne s'opère pas arbitrairement. L'historien se soumet à une échelle rigide de valeurs. Toujours, il respecte la vérité historique. C'est au cours de cette opération délicate que ses lecteurs et ses critiques se rendront compte si un historien est impartial ou s'il a des partis pris. M. Toynbee, lui, ignore délibérément les faits qui contredisent les thèses qui lui tiennent à cœur ou qui le forceraient à nuancer ses affirmations. Le procédé est commode; il n'est pas recommandable.

16. Voir Charles A. Beard, "Review of A Study of History", American Historical Review, 40 (1935): 307-309, 45 (1940): 593-594; Lucien Febvre, "De Spengler à Toynbee: quelques philosophies opportunistes de l'histoire", Revue de Métaphysique et de Morale, 43 (1936): 573-602; Pieter Geyl, "Toynbee's System of Civilization", Journal of the History of Ideas, 9 (1948): 93-124; Gourou, "Civilisations et malchance géographique", loc. cit., 445-450; Lefebvre, "Compte rendu critique", loc. cit., 109-113; Sorokin, "Toynbee's Philosophy", loc. cit., 379-387; Lynn Thorndike, "Review of A Study of History", Journal of Modern History, 7 (1935): 315-317.

17. Henri-Irénée Marrou, "De la logique de l'histoire à une éthique de l'historien", Revue de Metaphysique et de Morale, 54 (1949): 248-249. 
M. Toynbee - ce qui est plus grave - nourrit des préjugés. L'une de ses bêtes noires c'est le nationalisme ${ }^{18}$. Après bien d'autres observateurs contemporains, il a constaté à quels abus et à quels excès l'idéal démocrato-nationaliste de la Révolution française et du XIXe siècle a conduit les hommes du XXe siècle. L'autarcie des pays industrialisés, la lutte impitoyable pour les marchés, le racisme de Hitler, le fascisme de Mussolini, le réveil de l'Asie, deux guerres mondiales, l'asservissement des peyples aux mystiques totalitaires, une Europe ravagée, un monde dG équilibré, telles sont, à première vue, quelques-unes des conséquences du culte nationaliste. Ce terrible bilan semble légitimer jusqu'à un certain point les dénonciateurs indignés du nationalisme. Cependant, ceux-ci ne doivent pas eux-mêmes sacrifier à l'idéologie qu'ils condamnent si éloquemment. Or, les critiques de $M$. Toynbee ont démontré que celui-ci est beaucoup trop indulgent envers le nationalisme impérialiste de sa mère-patrie. Lorsqu'il étudie la guerre des Boers, par exemple, il est facile de se rendre compte qu'il réagit comme le ferait un fidèle disciple de Cecil Rhodes. Les historiens Georges Lefebvre et Pieter Geyl lui ont justement reproché sa partialitét ${ }^{19}$. Ses préjugés antinationalistes le rendent injuste envers le Risorgimento italien qu'il a très mal interprété. ${ }^{20}$

L'horreur que M. Toynbee éprouve pour le nationalisme ne s'inspire pas uniquement de motifs désintéressés, humanitaires. Il est Anglais et Européen. A ce double titre, il peut difficilement approuver la révolte nationaliste des peuples encore hier docilement soumis à l'hégémonie des deux plus grandes puissances colonisatrices et impérialistes des temps modernes, la France et l'Angleterre. Le nationalisme des Boers, des Irlandais, des Slaves, des Arabes, des Chinois, des Hindous a définitivement rompu depuis une génération l'ancien équilibre qui avait permis à l'Europe occidentale de dominer le monde et à la Home Fleet d'établir la pax britannica aux quatre coins du globe.

18. Voir Study of History, édition abrégée, 285, 288-293, 314-315; édition complète, 1: 9, note (3); 4: 407-408, 543; Toynbee, "History Warns Modern Man", World Review (Mars 1949), 10.

19. Lefebvre, “Compte rendu critique", loc. cit., 113; Geyl, “Toynbee's System", loc. cit., 93-124. Voir ce qu'il dit des Boers dans Study of History, 4: 294-295.

20. Study of History, édition abrégée, 314. 
L'attitude de M. Toynbee à l'égard des États-Unis met très bien en lumière ce complexe de supériorité propre à l'Anglais et à l'Européen. La grande république américaine a reçu la lourde tâche de guider le monde atlantique. Ce phénomène de l'évolution historique blesse la susceptibilité de l'auteur de $A$ Study of History. Un historien anglo-canadien l'accuse d'avoir conservé tous les préjugés de l'époque victorienne au sujet des États-Unis. Il le soupçonne même d'avoir conclu à la décadence et au déclin de la civilisation occidentale tout simplement parce que pays est devenu, au cours du dernier quart de siècle, l'indigne héritier du pouvoir et du prestige qui hier appartenaient à l'Angleterre et à l'Europe de l'Ouest. ${ }^{21}$ Son nationalisme de Britisher et d'Européen en est humilié. Plusieurs Européens, qui gardent nostalgiquement le souvenir d'une époque glorieuse maintenant révolue, pensent comme $M$. Toynbee. Ce fait ne prouve pas leur objectivité, ni leur désintéressement. Encore moins leur humilité! Il est regrettable que l'auteur de Civilization on Trial entretienne de tels préjugés.

A Study of History, qui se veut une œuvre de synthèse, n'est qu'une brillante dissertation. Véritable jongleur, l'érudit M. Toynbee, à l'aide de la méthode comparative, manie avec une habileté bouleversante les faits, les accidents géographiques, les hommes, les événements qui ont rempli les annales de l'humanité depuis six mille ans. Lucien Febvre le compare à un "prestidigitateur"'22. Le mot n'est pas trop fort. Malheureusement, les études de M. Toynbee auront peut-être la conséquence néfaste de discréditer dans l'esprit des gens avertis et sérieux les véritables travaux de synthèse historique. Ceux-ci demeurent, néanmoins, le but ultime, le couronnement des recherches historiques. S'il en est ainsi, l'auteur de $A$ Study of History aura rendu un bien mauvais service à la science de l'histoire.

$$
\text { *** }
$$

Cet article s'intitule "Le métahistorien Arnold J. Toynbee et la minorité canadienne-française". Le lecteur qui a eu la patience

21. Underhill, "Arnold Toynbee, Metahistorian", loc. cit., 216.

22. Febvre, "De Spengler à Toynbee", loc. cit., 591. 
de nous suivre se demandera certainement pourquoi. Le moment est venu de répondre à cette question légitime.

La minorité canadienne-française n'a pas échappé à l'inventaire de $M$. Toynbee. Il parle de nous à quelques reprises ${ }^{23}$. Deux de ses déclarations ont surtout attiré l'attention. Dans son livre Civilization on Trial, il énonce avec l'autorité du prophète: "Si l'avenir de l'humanité dans un monde unifié est appelé, dans son ensemble, à être heureux, alors je prédirais que demain réserve un grand rôle dans l'ancien monde aux Chinois et, sur l'île de l'Amérique du Nord, aux Canadiens français. Quel que soit l'avenir du genre humain en Amérique du Nord, j'ai la conviction que ces Canadiens de langue française, en tout cas, prendront part aux derniers événements de l'histoire" ${ }^{24}$. Un article publié quelques mois plus tard démontre que le métahistorien n'a pas eu le temps de changer d'idée. "Aujourd'hui, y déclare-t-il, l'unité du monde peut fort bien être [ comme à d'autres époques] l'œuvre des habitants de pays qui n'ont pas eu un passé très glorieux. Si les grandes puissances s'entêtent à demeurer attachées à leur vieil idéal nationaliste - maintenant démodé, les peuples pour qui le nationalisme n'a pas été une heureuse expérience pourraient bien être les seuls capables d'apporter au monde la solution originale dont il a besoin. J'imagine que le peuple de l'avenir dans les Amériques pourrait bien être le peuple canadien-français; et en Asie, les Chinois." 25

Les Canadiens français appelés à réaliser l'unité du monde! Appelés du moins à fournir la solution originale qui amènerait cet heureux résultat! Prophétie propre à soulever l'enthousiasme et l'émotion. ${ }^{26}$ Réaction toute naturelle, quand on considère que tous

23. Study of History, 2: 71-72; 4: 163-165, 293, note (1); Civilization on Trial, 161; "History Warns Modern Man", loc. cit., 12.

24. Civilization on Trial, 161.

25. Toynbee, “History Warns Modern Man”, loc. cit., 12.

26. Voir Richard Arès, "Sur une prédiction d'Arnold Toynbee", loc. cit., 93-95; Jean-Paul Labelle, "La vitalité du Canada français", Relations, 10 (Mai 1950): 139; Firmin Létourneau, Histoire de l'Agriculture: Canada français (Montréal, 1950), 173. 
les peuples, même les moins doués, croient avoir une mission. Mais réfléchissons quelque peu; et surtout, soyons modestes. Nous avons, certes, maintenu, au prix de grands sacrifices, des traditions qui gardent une haute valeur chrétienne et humaniste. Cependant, d'autres peuples - ne l'oublions pas - en ont fait autant. Nous appartenons, par notre origine ethnique, à l'une des nations dont la contribution à la civilisation occidentale a été particulièrement importante. Nous-mêmes, n'avons-nous pas, jusqu'à nos jours, plutôt vécu des richesses culturelles accumulées par les autres? Dans les sciences, dans les arts, dans les lettres, dans le domaine de la pensée pure, nos œuvres ne sont pas si nombreuses. Le cardinal Villeneuve a déjà dressé un inventaire qui n'avait rien d'exaltant. La leçon d'humilité qui se dégage de cette magistrale conférence demeure toujours d'actualité27. Notre foi nous rattache a l'Église catholique qui, depuis sa fondation divine, a si vaillamment défendu, dans un monde trop souvent hostile ou indifférent, les vérités éternelles, seules capables de donner un sens à la vie terrestre. Nos missionnaires, nos communautés d'hommes et de femmes, notre clergé, nos institutions d'enseignement et d'hospitalisation, notre fidélité exemplaire à la foi ancestrale nous ont permis de contribuer largement et généreusement à l'œuvre de l'évangélisation et de la rédemption au milieu de nous et autour de nous. Et c'est peut-être là notre plus grand mérite. Toutefois rappelons-nous qu'il existe quelque trois cents autres millions de catholiques qui œuvrent aussi dans l'Église et dans le monde.

Quel a été et quel est notre rôle à l'échelle internationale? Les Canadiens français siègent rarement dans les conseils internationaux. La politique extérieure du pays ne dépend pas de nous. Cette affirmation ne surprendra personne. Ceux qui assistent aux grandes conférences mondiales à Paris, à San Francisco, à New York, y chercheraient en vain la voix du Canada français. Comment celui-ci réussira-t-il à faire accepter, même à faire connaître, sa solution originale qui établira l'unité du monde? M. Toynbee lui-même pourrait difficilement répondre à cette question.

27. Jean-Marie-Rodrigue Villeneuve, "L'Université, école de haut savoir et source de directives sociales", dans Quelques Pierres de doctrine (Montréal, 1938), 79-112. 
C'est à se demander si cet écrivain est sérieux. On peut en douter. Après avoir prédit aux Canadiens français une mission unique dans les siècles à venir, il avoue candidement qu'avant la fabrication de la bombe atomique il aurait assigné ce rôle aux Tibétains ou aux Esquimaux. Ces peuples jouissaient, dit-il, d'une très grande sécurité avant l'ère atomique. Maintenant, à cause de la rivalité russo-américaine, le Tibet et le grand nord canadien seraient destinés à devenir des thétres de guerre. Il a donc pensé à nous en désespoir de cause. Cependant, il semble que ce choix ne le satisfait pas outre mesure. En veine de confidences, M. Toynbee laisse clairement entendre que les nègres pygmées d'Afrique Centrale pourraient bien devenir les seuls gardiens et héritiers de la civilisation atlantique si le monde se fait atomiser. ${ }^{28}$ Nous aimons bien les Tibétains, les Esquimaux et les tribus de l'Afrique. Nous sommes même prêts à leur reconnaître toutes sortes de qualités et de mérites. Mais, tout de même... M. Toynbee revient sur la même idée lorsqu'il souligne, dans l'article où il s'intéresse encore à nous, que les nègres américains, les réfugiés des camps européens, les peuples mal partagés d'Afrique et d'Asie offrent des ressources d'énergies encore inutilisées. "Peutêtre, suggère-t-il, que ces peuples seront nos sauveurs." ${ }^{29}$ Personne n'y verra objection. Cependant, le lecteur le plus indulgent de M. Toynbee constatera, bien timidement, que l'auteur semble embrouille dans ses cartes. Les cartomanciennes pratiquent aussi cet art de prédire plusieurs choses à la fois. Inévitablement, une ou deux de leurs prédictions courent la chance de se réaliser.

Est-il possible de savoir pourquoi le métahistorien Toynbee nous apprécie tant? Cet ardent admirateur de l'empire britannique et ce contempteur du nationalisme contemporain nous considère comme le peuple colonial parfait. Nous avons, selon lui, accepté la conquête anglaise avec satisfaction, sinon avec enthousiasme. ${ }^{30}$ Notre nationalité n'a jamais connu les succès qui enivrent. ${ }^{31}$ Notre passé ne nous rappelle aucune réalisation glorieuse qui nous rendrait infatués

28. Voir Civilization on Trial, 161-162.

29. Toynbee, "History Warns Modern Man", loc. cit., 13.

30. Voir Study of History, 4: 163-165.

31. Toynbee, “History Warns Modern Man”, loc. cit., 11. 
de nous-mêmes. ${ }^{32}$ Contrairement aux Irlandais qui ont conservé "l'obsession de leur passé, qui a fait le désespoir des hommes d'État britanniques" ${ }^{33}$, nous avons, toujours d'après M. Toynbee, manifesté à un degré étonnant ce qu'il appelle la "plasticité psychologique [ des peuples coloniaux ] qui sert si puissamment à la consolidation politique" ${ }^{34}$. L'auteur insiste beaucoup sur cette "plasticité psychologique" des populations coloniales qui a favorisé l'expansion des grandes puissances impérialistes, en particulier l'Angleterre. ${ }^{35}$ Parce que nous avons pratiqué depuis la conquête un nationalisme timide, incomplet, nous avons permis à la politique britannique de réaliser, au Canada, l'un des "plus grands triomphes" 36 de son expansion coloniale. Si tous les groupes minoritaires, si toutes les nationalités conquises, si tous les peuples colonisés avaient témoigné la docilité, la soumission, la "plasticité psychologique" des Canadiens français, le bel équilibre du XIXe siècle, sans aucun doute, se serait maintenu au profit des anciennes puissances impérialistes. Pour réaliser l'unité du monde, il faudra que toutes les nations renoncent, au nom de l'idéal chrétien de la fraternité humaine et en faveur d'un gouvernement supra-national, à l'autonomie que chacune d'entre elles défend si jalousement et si égoïstement. M. Toynbee juge que notre exemple devrait les inspirer. Tels sont nos mérites, nos principaux mérites, aux yeux de l'auteur de $A$ Study of History. Devons-nous nous féliciter de son admiration pour la minorité canadienne-française? Chacun demeure libre de répondre selon ses convictions personnelles.

Université de Montreal.

Michel Brunet

32. Ibid., 12.

33. Study of History, 4: 292.

34. Ibid., 3: 303, note (1).

35. Ibid., 4: 292-293; du même auteur, The Conduct of the British Emirize. Foreign Relations since the Peace Settlement (Londres, 1928), 37-38.

36. Study of History, 4: 292. M. Toynbee considère l'Union de l'Afrique du Sud comme le second succès remarquable de la politique britannique d'expansion in périaliste. Il écrivit ces pages avant la victoire du parti nationaliste Boer. 